\title{
Relationship between X-ray and ultraviolet emission of flares from dMe stars observed by XMM-Newton
}

\author{
U. Mitra-Kraev ${ }^{1}$, L. K. Harra ${ }^{1}$, M. Güdel ${ }^{2}$, M. Audard ${ }^{3}$, G. Branduardi-Raymont ${ }^{1}$, H. R. M. Kay ${ }^{1}$, R. Mewe ${ }^{4}$, \\ A. J. J. Raassen ${ }^{4,5}$, and L. van Driel-Gesztelyi ${ }^{1,6,7}$ \\ 1 Mullard Space Science Laboratory, University College London, Holmbury St. Mary, Dorking, Surrey RH5 6NT, UK \\ e-mail: umk@mssl.ucl.ac.uk \\ 2 Paul Scherrer Institut, Würenlingen \& Villigen, 5232 Villigen PSI, Switzerland \\ 3 Columbia Astrophysics Laboratory, Columbia University, 550 West 120th Street, New York, NY 10027, USA \\ ${ }^{4}$ SRON National Institute for Space Research, Sorbonnelaan 2, 3584 CA Utrecht, The Netherlands \\ 5 Astronomical Institute "Anton Pannekoek", Kruislaan 403, 1098 SJ Amsterdam, The Netherlands \\ ${ }^{6}$ Observatoire de Paris, LESIA, 92195 Meudon, France \\ 7 Konkoly Observatory, 1525 Budapest, Hungary
}

Received 30 April 2004 / Accepted 30 September 2004

\begin{abstract}
We present simultaneous ultraviolet and X-ray observations of the dMe-type flaring stars AT Mic, AU Mic, EV Lac, UV Cet and YZ CMi obtained with the XMM-Newton observatory. During $40 \mathrm{~h}$ of simultaneous observation we identify 13 flares which occurred in both wave bands. For the first time, a correlation between X-ray and ultraviolet flux for stellar flares has been observed. We find power-law relationships between these two wavelength bands for the flare luminosity increase, as well as for flare energies, with power-law exponents between 1 and 2. We also observe a correlation between the ultraviolet flare energy and the X-ray luminosity increase, which is in agreement with the Neupert effect and demonstrates that chromospheric evaporation is taking place.
\end{abstract}

Key words. stars: chromospheres - stars: coronae - stars: flare - stars: late-type - ultraviolet: stars - X-rays: stars

\section{Introduction}

XMM-Newton is an ideal platform for observing cosmic sources simultaneously in X-rays and ultraviolet (UV). In particular, flux and energy comparisons between these wavebands are important in understanding the flare mechanisms in stellar coronae. Late-type stars typically show high coronal activity similar to the Sun, with flux variability through all observed wavelength bands. dMe-type stars are particularly active. They show frequent flaring activity (Pallavicini et al. 1990), as well as strong emission lines (including the $\mathrm{H} \alpha$ Balmer emission line, which is denoted by the "e" in dMe). Soft X-rays $(<12 \mathrm{keV})$ typically originate in the corona, while $\mathrm{UV}$ radiation comes from the chromosphere and transition region. Flares are observed in both wave bands, but what is the physical process that connects X-ray and UV flares?

In the chromospheric evaporation picture (see e.g. Antonucci et al. 1984), flares are believed to be due to the energy release from the reconnection of magnetic field lines in the lower corona. Electrons are accelerated at the reconnection site. They gyrate downward along the magnetic field lines, emitting gyrosynchrotron radio emission. Their collision with denser material in the chromosphere unleashes bremsstrahlung seen in hard X-rays $(>20 \mathrm{keV})$. Both the gyrosynchrotron radio and hard X-ray flare emissions are impulsive, with a fast increase and a steep decay. At the same time, the electrons impulsively heat the chromosphere, which results in prompt optical/UV emission which closely correlates with the hard X-ray emission (Hudson et al. 1992). During the collisions, the chromospheric ions get further ionised, the material heats up and evaporates, increasing the density and the temperature of the reconnected loops in the corona. The hot material is seen in soft X-ray and extreme ultraviolet emissions, where the light curves are more gradual, with a slower increase than the impulsive emission and a much longer, exponential decay. The impulsive and gradual emissions are often temporally connected through the "Neupert effect" relation (Neupert 1968; Dennis \& Zarro 1993)

$L_{\text {grad }}(t)=q \cdot \int_{0}^{t} L_{\text {impuls }}\left(t^{\prime}\right) \mathrm{d} t^{\prime}$

indicating that the gradual radiative loss rate is directly proportional to the cumulative impulsive energy input, and suggesting that the energy input from the non-thermal electrons is responsible for the heating of the plasma. 
Table 1. Observational parameters and results.

\begin{tabular}{cccccc}
\hline \hline & EV Lac & UV Cet & YZ CMi & AU Mic & AT Mic \\
\hline Index & $\mathrm{A}$ & $\mathrm{B}$ & $\mathrm{C}$ & $\mathrm{D}$ & $\mathrm{E}$ \\
Spectral type & $\mathrm{dM} 4.5 \mathrm{e}$ & $\mathrm{dM} 5.5 \mathrm{e}+\mathrm{dM} 5.5 \mathrm{e}$ & $\mathrm{dM} 4.5 \mathrm{e}$ & $\mathrm{dM} 1 \mathrm{e}$ & $\mathrm{dM} 4.5 \mathrm{e}+\mathrm{dM} 4.5 \mathrm{e}$ \\
Distance $d_{\star}[\mathrm{pc}]$ & $5.0^{a}$ & $2.6^{b}$ & $5.9^{a}$ & $9.9^{a}$ & $10.2^{a}$ \\
UV filter & $U V W 1$ & $U V W 1$ & $U V W 2$ & $U V W 2$ & $U V W 2$ \\
UV range $[\AA]$ & $2450-3200$ & $2450-3200$ & $1800-2250$ & $1800-2250$ & $1800-2250$ \\
\hline $\bar{L}_{\text {uv }}\left[10^{29} \mathrm{erg} / \mathrm{s}\right]$ & 0.434 & 0.0258 & 1.28 & 12.3 & 6.39 \\
$\bar{L}_{\mathrm{x}}\left[10^{29} \mathrm{erg} / \mathrm{s}\right]$ & 0.545 & 0.0333 & 0.370 & 2.99 & 2.89 \\
Time-lag UV-X-ray $[\mathrm{s}]$ & $-600_{-600}^{+600}$ & $-200_{-200}^{+600}$ & $-600_{-400}^{+600}$ & $-400_{-400}^{+400}$ & $-1000_{-1000}^{+800}$ \\
X-ray/UV max. correlation & 0.68 & 0.90 & 0.78 & 0.68 & 0.84 \\
\hline
\end{tabular}

${ }^{a}$ Perryman et al. (1997).

${ }^{b}$ Harrington \& Dahn (1980).

The soft X-rays originating from the hot ( $>1 \mathrm{MK})$ coronae of late-type stars are dominated by emission lines $(\mathrm{H}-$ and $\mathrm{He}$-like transitions of $\mathrm{C}, \mathrm{N}, \mathrm{O}, \mathrm{Ne}, \mathrm{Mg}$, and $\mathrm{Si}$, and $\mathrm{Fe} \mathrm{K}-$ and L-shell transitions), indicating thermal processes. The UV emission from dMe stars is mainly line emission formed around $10^{4} \mathrm{~K}$ (Linsky et al. 1982), though during flares continuum emission is also observed in the near-optical UV band (Hawley \& Pettersen 1991). At optical wavelengths, both impulsive continuum and gradual line emission are observed during flares (García-Alvarez et al. 2002). The Neupert effect has been known to exist between impulsive U-band and soft X-ray emission (Güdel et al. 2002; Hawley et al. 1995).

Previous investigations relating UV and soft X-rays in late-type stars were either focused on individual flares or statistical flux-flux relationships for the entire stellar emission with no temporal resolution. The UV data were collected with the International Ultraviolet Explorer (IUE), which obtained low dispersion spectra in the 1150-3200 $\AA$ band, and compared with data from the X-ray satellites Einstein $(0.2-4 \mathrm{keV})$, EXOSAT (0.06-2 keV) and ROSAT (0.1-2.4 keV), and more recently UV data from the Hubble Space Telescope were compared with X-ray data from the Chandra satellite (Ayres et al. 2001). To distinguish flaring from non-flaring conditions, Mathioudakis \& Doyle (1989) compared flux-flux relationships of inactive $\mathrm{dM} / \mathrm{dK}$ to active $\mathrm{dMe} / \mathrm{dKe}$ stars, using the latter as a proxy for flaring conditions. They find a power-law relationship with a slope of $\approx 1$ between chromospheric Mg II $\mathrm{h}$ and k (2795-2803 $\AA$ ) IUE and coronal X-ray (Einstein and EXOSAT) flux, but only for the active stars. The inactive stars are scattered, with generally lower $\mathrm{X}$-ray luminosity. Thus, the coronal X-ray emission is enhanced for stars with flares compared to stars without. Similar analyses for F-K type stars have been made by Schrijver et al. (1992, including a basal flux subtraction) and Ayres et al. (1995, fluxes scaled to bolometric fluxes), who found power-law slopes between 1.5 and 2.9. None of these papers tested the relationship of different fluxes for individual flares.
We set out to probe directly the statistical flux-flux relationship between X-ray and UV emission for flares. dMe-type stars are the natural choice, as they flare frequently. The instruments on-board XMM-Newton provide an excellent opportunity for carrying out these observations.

This paper is structured in the following way: Sect. 2 describes the observations and the instrumental setup. The results are presented in Sect. 3, starting with the X-ray and UV luminosity light curves of EV Lac, UV Cet, YZ CMi, AU Mic and AT Mic. The respective light curves are cross-correlated and flares identified in both wavebands. Then, the power-law relationships between luminosity increase per flare and flare energy are presented. We discuss the results in Sect. 4 and conclude with Sect. 5.

\section{Observations}

We present XMM-Newton data of dMe-type stars obtained during the Reflection Grating Spectrometer (RGS) Guaranteed Time Programme. We make use of the pn-European Photon Imaging Camera (EPIC-pn, Strüder et al. 2001), and the Optical Monitor (OM, Mason et al. 2001). The EPIC-pn covers the range $0.2-12 \mathrm{keV}(1-62 \AA)$. The OM observed with two different UV filters, one for each observation, namely the $U V W 1(2450-3200 \AA)$ and the $U V W 2(1800-2250 \AA)$ filter.

Table 1 gives a list of the targets, shows the spectral type, the distance to the star (from SIMBAD parallaxes), the observed UV range and UV filter. The index is for further reference in the later plots. While some of the X-ray results of these targets have already been published (Raassen et al. 2003; Ness et al. 2003; Magee et al. 2003), this is the first time that we make use of the simultaneously obtained UV data.

For all observations, OM observed in IMAGE mode, where a single exposure lasted $800 \mathrm{~s}$ and the dead-time between two exposures was $320 \mathrm{~s}$, thus resulting in a time resolution of $1120 \mathrm{~s}$. The data for the EPIC-pn light curves were binned into $200 \mathrm{~s}$ time intervals. All data were reduced with the XMM-Newton Science Analysis System (SAS) version 5.4. 

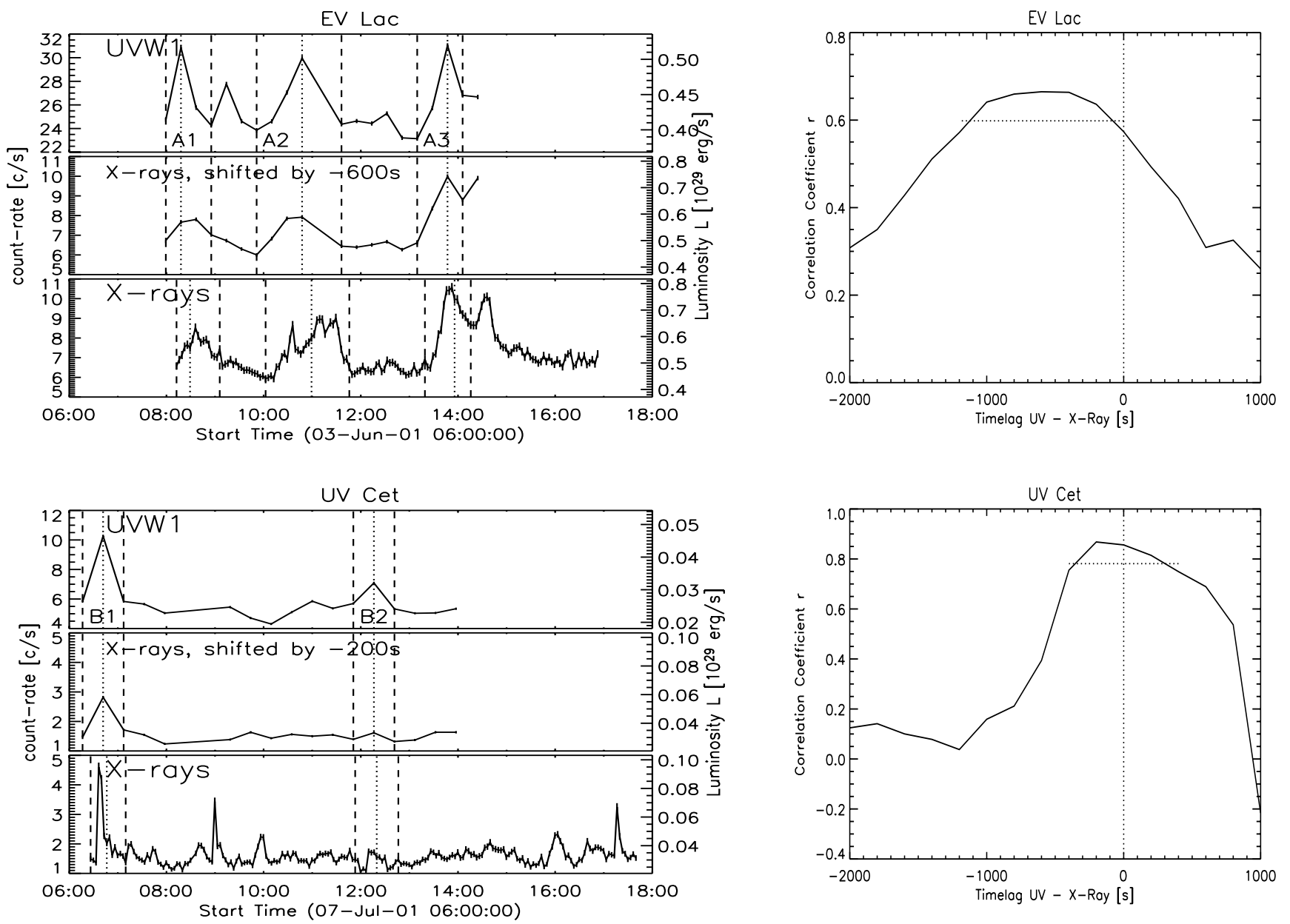

Fig. 1. The light curves (left panels) and their cross-correlations (right panels) of EV Lac and UV Cet, where the $U V W 1$ filter for the OM was used. The lowest panel in each left graph shows the 200s-binned X-ray data, the upmost panel the UV light curve, and the middle panel the X-ray light curve at maximum correlation (shifted by the time lag) and binned to UV resolution ( $800 \mathrm{~s}$ integration time and a cadence of $1120 \mathrm{~s}$ ). The count rate is given on the left, the luminosity on the right ordinate. The dashed vertical lines indicate the start and the end of the flare, the dotted vertical lines the time of the flare peak in the UV. The right panels show the cross-correlation between the X-ray and UV light curve for each target (see main text). The horizontal dotted lines are at $90 \%$ level of each maximum and mark the peak error interval.

\section{Results}

\subsection{Light curves}

\subsubsection{Determining the luminosity}

Figures 1 and 2 (left panels) display the X-ray and UV light curves of EV Lac, UV Cet, YZ CMi, AU Mic and AT Mic. In the ultraviolet, EV Lac and UV Cet (Fig. 1) were observed with the $U V W 1$ filter, whereas YZ CMi, AU Mic and AT Mic (Fig. 2) were observed with the $U V W 2$ filter.

The conversion from X-ray and UV count rates to luminosity has been made in the following way: the UV luminosity $L_{\mathrm{uv}}$ is given by

$L_{\mathrm{uv}}=4 \pi d_{\star}^{2} \cdot G \cdot \Delta_{\mathrm{uv}} \cdot c_{\mathrm{uv}}$,

with $c_{\mathrm{uv}}$ the UV count rate and $d_{\star}$ the distance to the star (see Table 1). $G$ is the factor to convert from count rate to flux, derived by folding stellar spectra with the in-flight response curves of the $\mathrm{OM}$ and tabulated on the XMM-Newton SAS homepage at Vilspa. The values of $G$ for M-type stars are $7.32( \pm 0.39) \times 10^{-16} \mathrm{erg} / \mathrm{cm}^{2} / \AA /$ count for the $U V W 1$ filter and
$1.04( \pm 0.21) \times 10^{-13} \mathrm{erg} / \mathrm{cm}^{2} / \AA /$ count for the $U V W 2$ filter. $\Delta_{\mathrm{uv}}$ is the bandwidth of the UV filter, $750 \AA$ for $U V W 1$ and $450 \AA$ for $U V W 2$. The values of the average stellar UV luminosities over the whole observations, $\bar{L}_{\mathrm{uv}}$, are displayed in Table 1 .

For the X-ray luminosity, the average luminosity $\bar{L}_{\mathrm{x}}$ has been obtained from fitting the EPIC-pn spectrum from the whole observation with a 3-Temperature CIE (collisional ionisation equilibrium) model within the SPEX package (Kaastra et al. 2002) with variable elemental abundances. The precise values of the abundances do not matter in this context, as the value for the average luminosity, which is directly given by the count rate in the spectrum, is stable. The value of $\bar{L}_{\mathrm{x}}$ for each target, which includes dead-time corrections, is displayed in Table 1 . The three temperatures are typically 2-3 MK, 7-8 MK and $>20 \mathrm{MK}$. The X-ray luminosity $L_{\mathrm{x}}$ is then given by the average luminosity $\bar{L}_{\mathrm{x}}$ divided by the average count rate $\bar{c}_{\mathrm{X}}$ times the X-ray count rate $c_{\mathrm{X}}$

$L_{\mathrm{x}}=\frac{\bar{L}_{\mathrm{x}}}{\bar{c}_{\mathrm{x}}} \cdot c_{\mathrm{x}}$ 

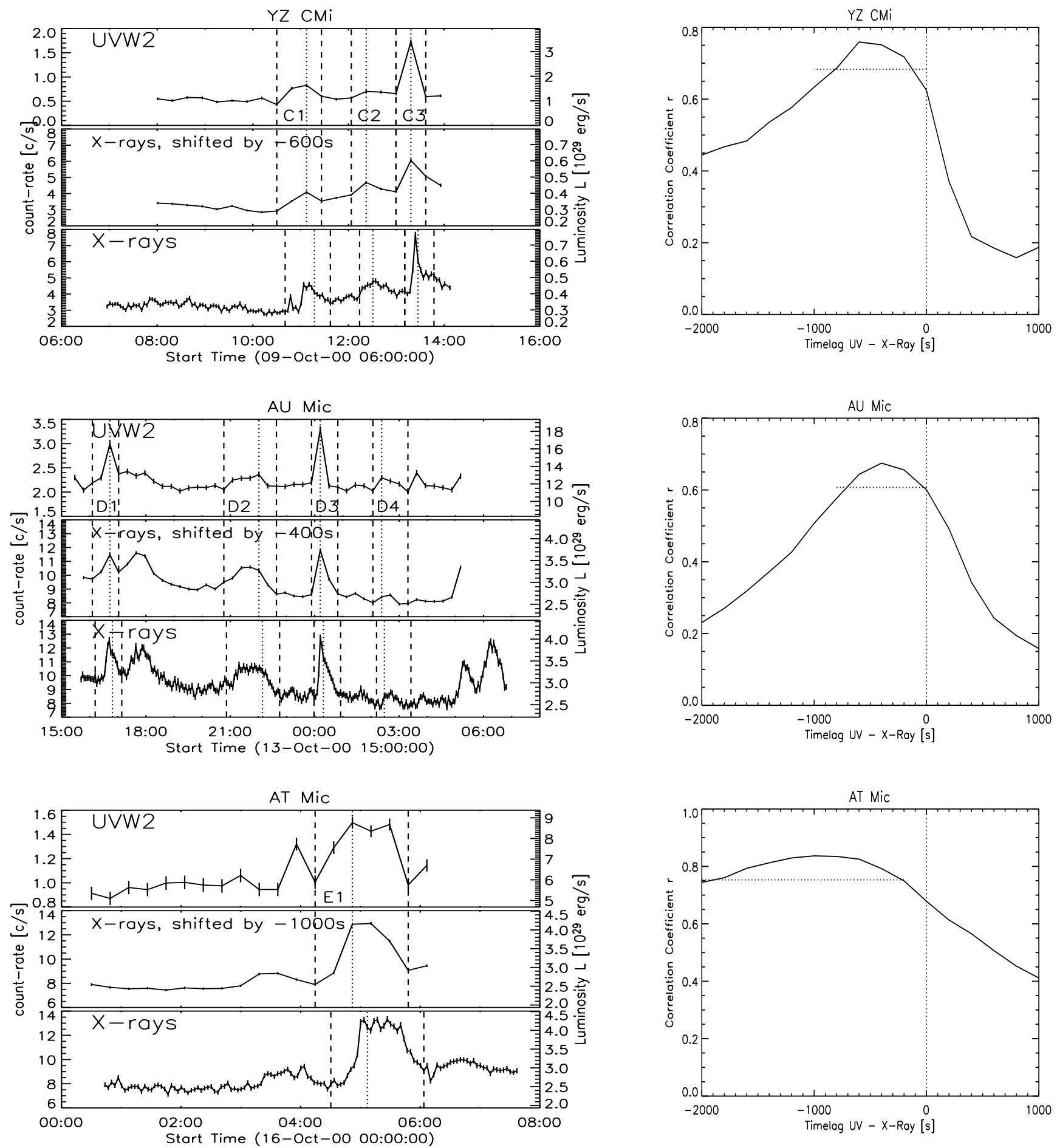

Fig. 2. The light curves (left panels) and their cross-correlations (right panels) of YZ CMi, AU Mic and AT Mic, where the $U V W 2$ filter for the OM was used. The same explanations as in Fig. 1 hold.

One might argue that the different shapes of the quiescent and flaring X-ray spectrum have a non-linear effect on the scaling of the luminosity. The $\mathrm{X}$-ray spectrum is dominated by lines in the range of $0.2-1 \mathrm{keV}$ and has a decreasing exponential tail above $1 \mathrm{keV}$. During flares, the exponential tail falls off more slowly than during quiet times, indicating higher temperatures and energies, while the low-energy range rises uniformly. Our fits show that most of the energy comes from the low-energy range. The values for the total observations are $0.2-1 \mathrm{keV} \sim$ $70-75 \%, 1-2 \mathrm{keV} \sim 15-20 \%$, and $2-12 \mathrm{keV} \sim 10 \%$, while during flares the contribution to the lowest energy band is reduced by about $5 \%$, and increased by about $5 \%$ for the highest energy band. This suggests that the approximation that the luminosity scales linearly with the count rate is a fair assumption. 


\subsubsection{Cross-correlation between the UV and X-ray flux}

The two light curves from each of the five observations show a good correlation. For an increase in the UV, we generally also see an increase in the X-rays. Each pair of light curves has been cross-correlated in such a way that the 200s-binned X-ray light curve (lowest panels in the light curve plots of Figs. 1 and 2) was shifted by a multiple of $200 \mathrm{~s}$, then binned to the UV resolution and correlated with the UV light curve (upmost panel). The plots on the right hand side of Figs. 1 and 2 show the crosscorrelation for each target. The abscissa displays the time-lag between the UV and X-ray light curves, the ordinate the corresponding correlation coefficient. For all observations, the function has a distinct peak with a maximum correlation coefficient between 0.68 and 0.90 . For the error estimate in time, the width of the cross-correlation distribution function at the $90 \%$ level of the correlation coefficient maximum was calculated (horizontal dotted lines in the cross-correlation plot). This error corresponds to about the $1 \sigma$ error of the Fisher $z$-test. In all but the UV Cet observation, which is dominated by short X-ray flares which are below the OM resolution, the entire peak within the error lies left of zero, indicating that flares tend to peak earlier in UV than in X-rays. The middle panels of the light curve plots show the X-ray light curves at maximum correlation (shifted by the time-lag) and binned to UV resolution.

\subsubsection{Flare identification}

In both the UV and the X-ray (low 800 s-resolution) light curves (upper two panels in Figs. 1 and 2) flares are identified. The flare criteria are: (i) it has to appear in both wave bands simultaneously. (ii) The difference between flare peak and start intensity exceeds $3 \sigma$ of the noise ( $3 \times$ average noise). (iii) The difference between flare peak and end intensity exceeds $3 \sigma$ in at least one of the light curves (and $1 \sigma$ in the other). The beginnings and ends of the flares are marked with a dashed vertical line, the flare peak with a dotted vertical line. Each flare is named by a letter and a number displayed under the UV light curve.

\subsection{The UV-X-ray relationship in flares}

For each flare, the background-subtracted energy from flare start to end $E_{\mathrm{f}}=\int_{f_{\text {start }}}^{f_{\text {end }}}\left(L(t)-L_{\mathrm{f}}^{\min }\right) \mathrm{d} t$ as well as the luminosity increase from flare start to flare peak have been obtained for both UV and X-rays (low 800 s-resolution). Figure 3 shows the relationship between UV and X-rays for the observed flares from EV Lac and UV Cet ( $U V W 1$ filter, left side) and from YZ CMi, AU Mic and AT Mic ( $U V W 2$ filter, right side). The upper panels are for the luminosity, the lower panels for the energy relationship. We define the spectral luminosity density (luminosity per unit wavelength) $\mathcal{L}:=L / \Delta$ and the spectral energy density $\mathcal{E}:=E / \Delta$, with $\Delta$ being the width of the respective passband. The flare data are fitted with a power-law

$(\mathrm{X}$-ray $)=10^{c} \cdot(\mathrm{UV})^{k}$,
Table 2. Relationship parameters for $\log (\mathrm{X}$-ray $)=\kappa \cdot \log (\mathrm{UV})+c$.

\begin{tabular}{cccc}
\hline \hline & & $\kappa$ & $c$ \\
\hline \multirow{2}{*}{$\mathcal{L}$} & $U V W 1$ & $1.17 \pm 0.05$ & $-3.1 \pm 1.4$ \\
& $U V W 2$ & $1.10 \pm 0.09$ & $-2.5 \pm 2.5$ \\
\hline \multirow{2}{*}{$\mathcal{E}$} & $U V W 1$ & $1.21 \pm 0.05$ & $-4.5 \pm 1.4$ \\
& $U V W 2$ & $1.68 \pm 0.13$ & $-20 \pm 4$ \\
\hline
\end{tabular}

the values of $c$ and $\kappa$ are given in Table 2. The regressions have been made in the following way: for each regression, we have first applied two linear ordinary least-square (OLS) fits (Isobe et al. 1990) to the logarithmic data, one for $Y$ dependent on $X$, taking the $Y$-error into account, the other for $X$ dependent on $Y$, taking the $X$-error into account (linfit.pro in IDL). Given the two regressions (slopes $\beta_{1}$ and $\beta_{2}$ ), the bisector was calculated (slope $\beta_{3}=\tan \frac{\operatorname{atan} \beta_{1}+\operatorname{atan} \beta_{2}}{2}$ ) and the new variances obtained from Monte-Carlo simulations. The OLS bisector is thus chosen as the optimal regression.

For the luminosity (Eqs. (2) and (3)), we only considered the error from the counts, which basically follows Poisson statistics. Three further errors of the quantities used to derive the luminosities do not contribute much to the overall result. The uncertainty in stellar distance $(<5 \%)$ affects UV and X-ray luminosity alike and therefore does not influence the powerlaw slope. The $G$-factor of Eq. (2) carries an error of $5 \%$ for $U V W 1$ and $20 \%$ for $U V W 2$. As our targets all have similar spectral types, the possible systematic error is unlikely to affect the power-law exponent either. The third error is the (small) nonlinear contribution from the determination of the X-ray luminosity, which has been neglected (see Sect. 3.1.1).

\section{Discussion}

Comparing the peak luminosity increase as well as the total energy of flares, we find that the UV and X-ray emissions are correlated and that the relationships follow power laws, with power-law indices between 1 and 2 . For the $U V W 2$ results, the different power-law indices for the luminosity and energy relationships indicate that different time scales are involved in the energy release of X-ray and UV emission. While the luminosity increase roughly scales with an index of unity, the energy, which is time-integrated luminosity, scales with a power-law index significantly larger than unity. This suggests that for larger flares the duration of the X-ray emission is relatively longer than the duration of the UV emission. We are careful not to draw quantitative conclusions for the $U V W 1$ results because of the extremely small statistical sample.

The Neupert effect (Eq. (1)) predicts a temporal correlation between UV energy and X-ray luminosity. Although our data do not have a high enough temporal resolution, we can still test for a consistency with the Neupert effect indirectly. If the Neupert effect holds, then the total UV flare energy should be roughly proportional to the X-ray peak luminosity increase. From the chromospheric evaporation scenario, we indeed expect that a larger influx of non-thermal energy results in a larger amount of hot plasma, i.e. in more X-ray emission. We test for such a correlation, which is plotted in Fig. 4, and 

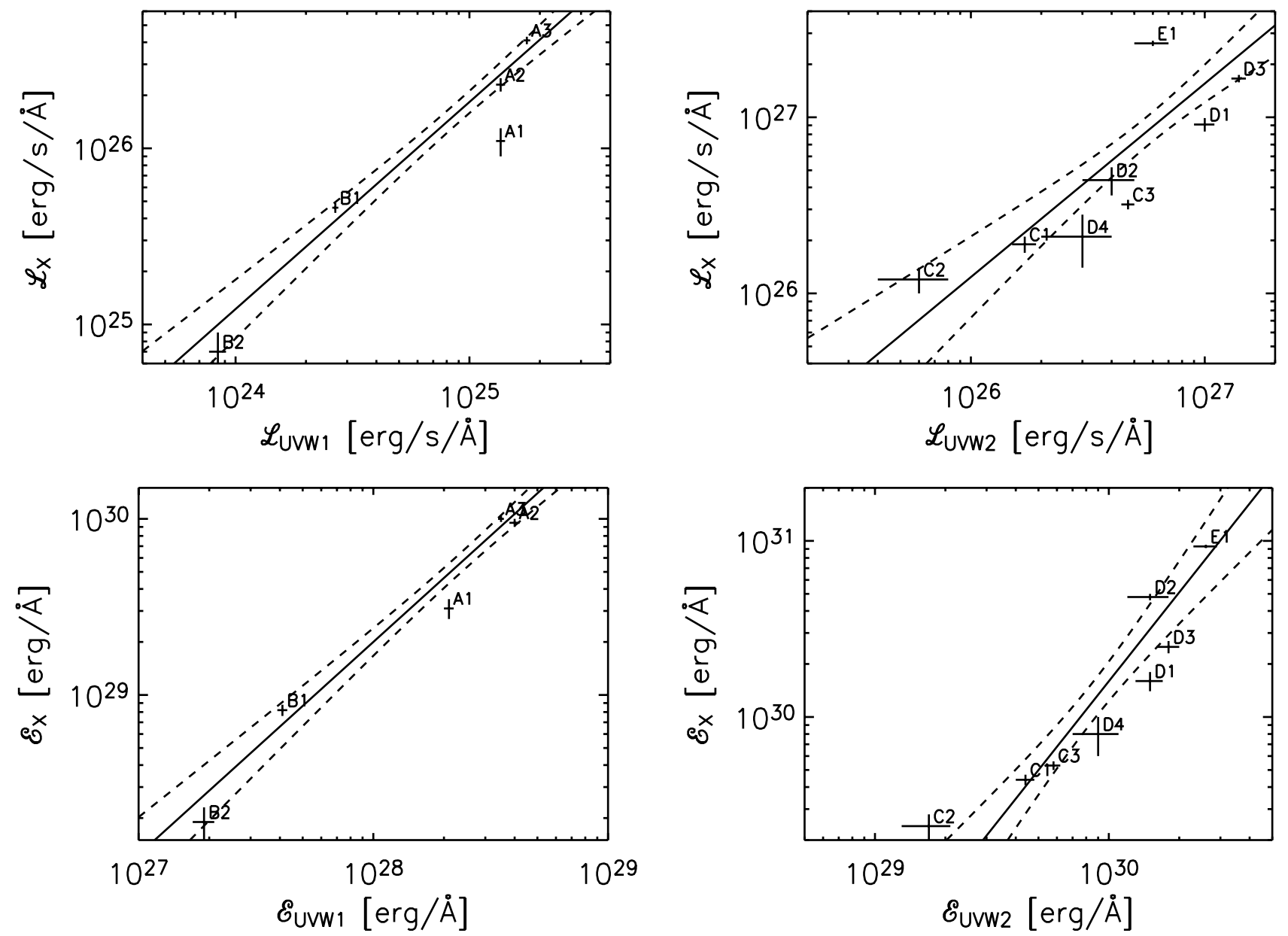

Fig. 3. X-ray vs. UV flare relationships. The two panels on the left use the $U V W 1$, the ones on the right the $U V W 2$ filter. The upper panels are for the luminosity increase, the lower panels for the flare energy. Used throughout for the regression are spectral luminosity density and spectral energy density, as defined in Sect. 3.2. The crosses denote the flares, their sizes corresponding to the $1 \sigma$ error. They are labelled according to their identification as in Figs. 1 and 2. The solid line in the plot indicates the best fit power-law regression. The flares have been weighted with their individual errors for the regression. The dashed lines contour the $3 \sigma$ error, where $\sigma$ is the standard deviation of the mean. The regression values are given in Table 2 .

the power-law fit parameters are given in Table 3. We find that there is such a correlation between the UV and the X-ray emission. The existence of an X-ray-luminosity/UV-energy correlation is in agreement with the Neupert-effect relationship and would suggest that the plasma is heated from the bottom of the magnetic flux tube to the top, first reaching the chromosphere and only a few hundred seconds later the hotter corona, which is consistent with chromospheric evaporation. It does not, however, confirm the Neupert effect. For that, the time resolution would have to be much better. The power-law exponent is close to unity for the $U V W 1$ flares and somewhat larger for the $U V W 2$ flares. If this correlation were indeed due to the Neupert effect, a power-law exponent of 1 would indicate that the factor of proportionality $q$ in Eq. (1) would be similar for all flares and thus imply similar physical conditions (e.g. the physics of energy transport and transformation) for all flares. A power-law exponent of a different value would suggest an energy dependence of $q$.

We would like to remark here that time resolution of the light curve also has an effect on the slope. While the flare
Table 3. Neupert relationship parameters: $\mathcal{L}_{\mathrm{x}}=10^{c} \cdot \mathcal{E}_{\mathrm{uv}}^{\kappa}$.

\begin{tabular}{ccc}
\hline \hline & $\kappa$ & $c$ \\
\hline$U V W 1$ & $1.08 \pm 0.05$ & $-4.4 \pm 1.5$ \\
$U V W 2$ & $1.40 \pm 0.11$ & $-15 \pm 3$ \\
\hline
\end{tabular}

energy is not strongly dependent on the bin size, the luminosity increases are. If the bin size is larger than the time scale of the flare, the underestimation of the luminosity increase can be severe. For X-rays, comparing the 200 s-binned data with the 800 s-binned data, we find that for small flares the luminosity increase is underestimated more than for large flares if the bins are large. For the UV flares, which are known to be more impulsive and have shorter time scales than the X-ray flares, the luminosity increase might be underestimated throughout. The luminosity-energy relationship is especially affected by this underestimation and the true correlation might be less steep and closer to unity. 

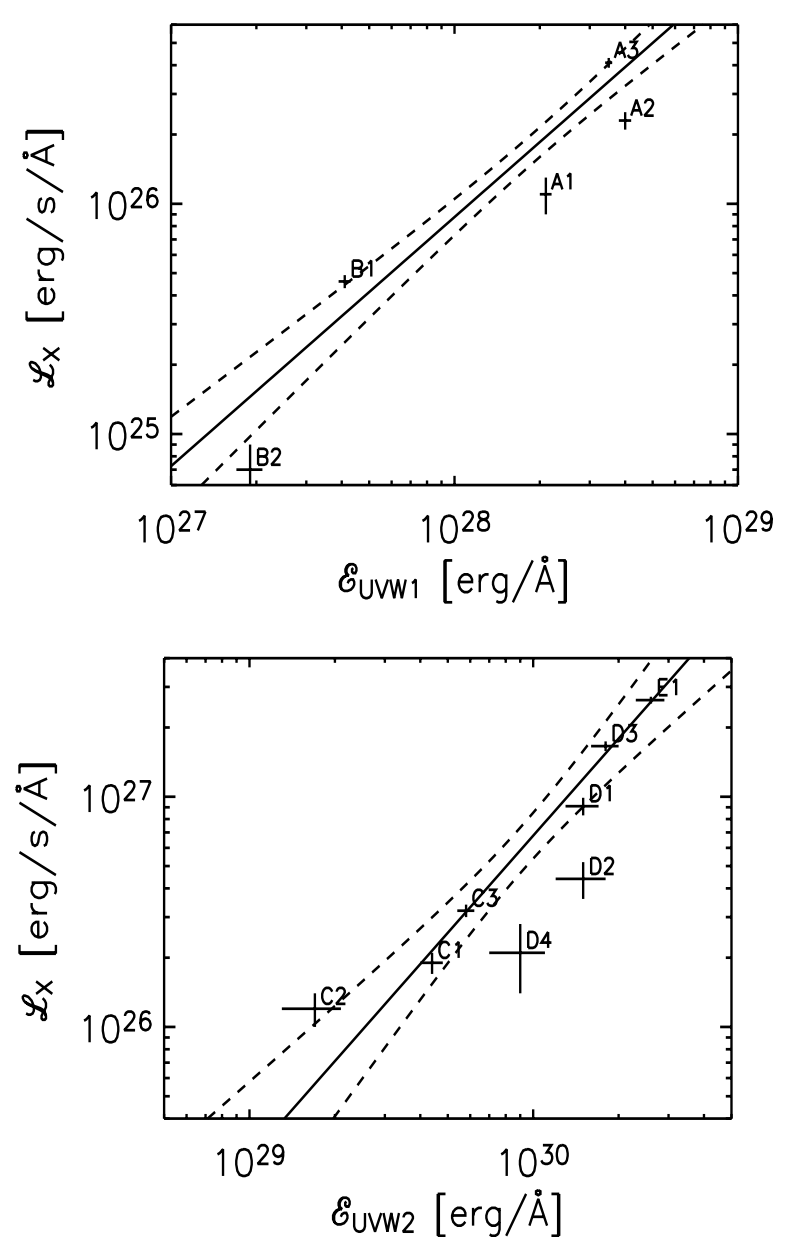

Fig. 4. Relationship between X-ray luminosity increase and UV flare energy. The conventions are the same as in Fig. 3. The fit parameters are given in Table 3 .

It may also be that all these correlations are just a manifestation of the big flare syndrome (BFS), which states that statistically all energetic flare phenomena are more intense in larger flares, regardless of the detailed physics (Kahler 1982). On the other hand, a correlation of two a priori unrelated parameters tells us something about the underlying physics. A correlation between extensive parameters (e.g. volume and mass) can be regarded as trivial, however, a correlation between intensive parameters (e.g. energy and luminosity) is not trivial. Since in this paper we are studying correlations between intensive parameters, even if the correlations are due to the BFS, they tell us something about the underlying physics. We can test for the BFS, if we compare the correlation coefficient of flare parameters we expect to correlate based on flare models, like $L_{\mathrm{x}}-L_{\mathrm{uv}}, E_{\mathrm{x}}-E_{\mathrm{uv}}$ and the relationship following from a possible Neupert effect $L_{\mathrm{x}}-E_{\mathrm{uv}}$, to flare parameters which have no physical reason to correlate $\left(E_{\mathrm{x}}-L_{\mathrm{uv}}\right)$. The correlation of the latter would reflect merely the BFS. The former being significantly higher than the latter would indicate an additional physical cause than just the BFS. Indeed, for the UVW2 filter, the relationship $E_{\mathrm{x}}-L_{\mathrm{uv}}$ gives a much lower correlation coefficient ( $r=0.63)$ than the ones which are physically related $(r=0.83$ for luminosity, $r=0.92$ for energy and "Neupert"). Therefore, the $U V W 2$ correlations are probably reflecting more than just the BFS. However, the correlation coefficients for the small $U V W 1$ sample are all above 0.95, including the BFS control parameters $E_{\mathrm{x}}-L_{\mathrm{uv}}$, and cannot distinguish between trivial and non-trivial scalings.

The slopes of the average stellar luminosities are close to unity, in agreement with Mathioudakis \& Doyle (1989). If the total stellar emission in X-rays and UV is a result of the superposition of many flares, then the X-ray to UV ratio of the average stellar luminosity should be similar to the X-ray to UV ratios of the flare energies. This is indeed so. For the flares, the ratio of X-ray to UV spectral energy density ranges from 10 to 29 (average of 19) for $U V W 1$ and from 0.9 to 3.6 (average of 1.7) for $U V W 2$. For the entire sample of stars, the ratio of $\mathrm{X}$-ray to UV average spectral luminosity density is between 15 and 16 for the $U V W 1$ filter and between 1.6 and 3.3 for the $U V W 2$ filter. The quiescent spectral luminosities have the same ratios. The energy ratios among the flares have a relatively wide spread because the power-law exponent is greater than unity. The stellar luminosity ratios within each UV wave band vary much less and are very close to the average flare energy ratios. Previously, Haisch et al. (1990) also reported that the ratio between energy losses in coronal X-rays and chromospheric Mg II lines is similar in flares and in quiescence in the dMe star Proxima Cen. This indicates a similar energy release physics both in flares and in the low-level emission. A possible explanation is that the low-level emission is in fact produced by a large number of unresolved flares that heat chromospheric gas and build up a corona by chromospheric evaporation. This hypothesis has recently found strong support from statistical light curve analysis (Güdel et al. 2003; Arzner \& Güdel 2004).

The extremely good temporal correlation between UV and X-ray flares is noteworthy. For almost every increase in UV emission, we also observe a corresponding increase in $\mathrm{X}$-rays, which is a similar result to the one found between the optical $U$-band and X-rays in a recent observation of Proxima Cen (Güdel et al. 2004). The overall good correlation between UV and X-ray flare occurrence is contrary to many previous observations, where some X-ray flares do not show a UV (or radio) counterpart and vice versa. This is especially true for older observations; this might be due to poorer instrumental performance. On the Sun, however, soft X-ray flares (usually less intense than the typical stellar flare observed here) only occasionally show simultaneous white-light emission, but all white-light flares also have a corresponding X-ray flare. Recent investigations by Matthews et al. (2003) show that white-light production is connected to peak pressure. It might be that in these more energetic stellar flare events such a critical peak pressure is almost always present, and therefore all flares are observed in both wavebands.

In the $U V W 2$ observations, we notice that there are two types of flares. The impulsive ones with a linear increase, an exponential decrease and a sharp peak, and the flares with a flat top. The latter are rising somewhat slower than the impulsive ones, showing prolonged, sustained peak emission. The tops of these flares (E1, D2, C2) are not constant, and in the case of E1 show periodic variations. We note that in the energy relationship plot these flat-top flares lie above the best-fit 
power-law correlation, which means that either there is a deficit in UV emission, or that there is an excess in X-rays. A similar phenomenon is seen in the correlation between microwave and hard X-ray emission in solar flares (Kosugi et al. 1988), where the long duration flares lie above the mean linear correlation, indicating a larger microwave to hard X-ray ratio for these flares.

\section{Conclusions}

We have investigated X-ray and UV light curves of several $\mathrm{dMe}$ stars, focusing on the temporal coincidence of flares and possible correlations between fluxes and radiative energies. The aim of our project was to study causal relations between mechanisms that produce UV and X-ray emission. The chromospheric evaporation scenario developed from solar observations and flare simulations predicts that the impulsive-phase optical/UV emission, most likely due to accelerated electrons impacting on the chromosphere, should precede the more slowly evolving soft X-ray emission that is emitted by the heated plasma. Specifically, the Neupert effect should approximately hold, i.e. the X-ray light curve is proportional to the time integral of the optical/UV light curve. Further, if the energy in the plasma stems from the accelerated electron population, we expect that the flare amplitudes are roughly correlated in amplitude or radiated energy.

We find evidence of both features predicted by the evaporation scenario. Firstly, most UV flares characteristically precede the X-ray peaks by typically ten minutes, which approximately coincides with the soft X-ray flare rise time. Secondly, we find a close near-linear correlation between the peak fluxes of the optical and the X-ray flares. The correlation becomes non-linear for the total radiative energies, implying that for larger flares the time scales of the X-ray flares become longer compared to the UV flare time scales.

An interesting aspect is the comparison of the X-ray to UV energy loss rate ratio of flares with the corresponding ratio between average emissions. We find that the two are indeed similar, which suggests that the total emission is a superposition of individual flare emissions. Our observations thus support a picture in which stellar chromospheres and coronae are continuously heated by impulsive energy release processes.

Acknowledgements. We kindly thank the anonymous referee for valuable comments and suggestions. U.M.K., L.K.H. and H.R.M.K. acknowledge financial support from the UK Particle Physics and Astronomy Research Council (PPARC). General stellar X-ray astronomy research at PSI has been supported by the Swiss National Science Foundation under project 20-66875.01. M.A. acknowledges support from NASA to Columbia University for XMM-Newton mission support and data analysis. Lv.D.G. acknowledges the Hungarian government grant OTKA T-038013.

In memory of Rolf Mewe, who sadly and unexpectedly passed away after this paper was submitted.

\section{References}

Antonucci, E., Gabriel, A. H., \& Dennis, B. R. 1984, ApJ, 287, 917

Arzner, K., \& Güdel, M. 2004, ApJ, 602, 363

Ayres, T. R., Brown, A., Osten, R. A., et al. 2001, ApJ, 549, 554

Ayres, T. R., Fleming, T. A., Simon, T., et al. 1995, ApJS, 96, 223

Dennis, B. R., \& Zarro, D. M. 1993, Sol. Phys., 146, 177

García-Alvarez, D., Jevremović, D., Doyle, J. G., \& Butler, C. J. 2002, A\&A, 383, 548

Güdel, M., Audard, M., Kashyap, V. L., Drake, J. J., \& Guinan, E. F. 2003, ApJ, 582, 423

Güdel, M., Audard, M., Reale, F., Skinner, S. L., \& Linsky, J. L. 2004, A\&A, 416, 713

Güdel, M., Audard, M., Skinner, S. L., \& Horvath, M. I. 2002, ApJ, 580, L73

Haisch, B. M., Butler, C. J., Foing, B., Rodonó, M., \& Giampapa, M. S. 1990, A\&A, 232, 387

Harrington, R. S., \& Dahn, C. C. 1980, AJ, 85, 454

Hawley, S. L., Fisher, G. H., Simon, T., et al. 1995, ApJ, 453, 464

Hawley, S. L., \& Pettersen, B. R. 1991, ApJ, 378, 725

Hudson, H. S., Acton, L. W., Hirayama, T., \& Uchida, Y. 1992, PASJ, 44, L77

Isobe, T., Feigelson, E. D., Akritas, M. G., \& Babu, G. J. 1990, ApJ, 364, 104

Kaastra, J. S., Mewe, R., \& Raassen, A. J. J. 2002, in ESA SP-488, New visions of the X-ray Universe in the XMM-Newton and Chandra Era, ed. F. Jansen

Kahler, S. W. 1982, JGR, 87, 3439

Kosugi, T., Dennis, B. R., \& Kai, K. 1988, ApJ, 324, 1118

Linsky, J. L., Bornmann, P. L., Carpenter, K. G., et al. 1982, ApJ, 260, 670

Magee, H. R. M., Güdel, M., Audard, M., \& Mewe, R. 2003, Adv. Space Res., 32, 1149

Mason, K. O., Breeveld, A., Much, R., et al. 2001, A\&A, 365, L36

Mathioudakis, M., \& Doyle, J. G. 1989, A\&A, 224, 179

Matthews, S. A., van Driel-Gesztelyi, L., Hudson, H. S., \& Nitta, N. V. 2003, A\&A, 409, 1107

Ness, J.-U., Schmitt, J. H. M. M., Audard, M., Güdel, M., \& Mewe, R. 2003, A\&A, 407, 347

Neupert, W. M. 1968, ApJ, 153, L59

Pallavicini, R., Tagliaferri, G., \& Stella, L. 1990, A\&A, 228, 403

Perryman, M. A. C., Lindegren, L., Kovalevsky, J., et al. 1997, A\&A, 323, L49

Raassen, A. J. J., Mewe, R., Audard, M., \& Güdel, M. 2003, A\&A, 411, 509

Schrijver, C. J., Dobson, A. K., \& Radick, R. R. 1992, A\&A, 258, 432

Strüder, L., Briel, U., Dennerl, K., et al. 2001, A\&A, 365, L18 